ARTICLE

https://doi.org/10.1038/s41467-019-14126-w

\title{
Direction and symmetry transition of the vector order parameter in topological superconductors $\mathrm{Cu}_{x} \mathrm{Bi}_{2} \mathrm{Se}_{3}$
}

T. Kawai ${ }^{1,4}$, C.G. Wang ${ }^{2,3,4}$, Y. Kandori ${ }^{1}$, Y. Honoki ${ }^{1}$, K. Matano ${ }^{1}$, T. Kambe ${ }^{1} \&$ Guo-qing Zheng $\oplus^{1,2 \star}$

Topological superconductors have attracted wide-spreading interests for the bright application perspectives to quantum computing. $\mathrm{Cu}_{0.3} \mathrm{Bi}_{2} \mathrm{Se}_{3}$ is a rare bulk topological superconductor with an odd-parity wave function, but the details of the vector order parameter d and its pinning mechanism are still unclear. Here, we succeed in growing $\mathrm{Cu}_{x} \mathrm{Bi}_{2} \mathrm{Se}_{3}$ single crystals with unprecedented high doping levels. For samples with $x=0.28,0.36$ and 0.37 with similar carrier density as evidenced by the Knight shift, the in-plane upper critical field $H_{\mathrm{c} 2}$ shows a two-fold symmetry. However, the angle at which the $H_{\mathrm{c} 2}$ becomes minimal is different by $90^{\circ}$ among them, which indicates that the d-vector direction is different for each crystal likely due to a different local environment. The carrier density for $x=0.46$ and 0.54 increases substantially compared to $x \leq 0.37$. Surprisingly, the in-plane $H_{c 2}$ anisotropy disappears, indicating that the gap symmetry undergoes a transition from nematic to isotropic (possibly chiral) as carrier increases.

\footnotetext{
${ }^{1}$ Department of Physics, Okayama University, Okayama 700-8530, Japan. ${ }^{2}$ Institute of Physics, Chinese Academy of Sciences, and Beijing National Laboratory for Condensed Matter Physics, 100190 Beijing, China. ${ }^{3}$ School of Physical Sciences, University of Chinese Academy of Sciences, 100190 Beijing, China. ${ }^{4}$ These authors contributed equally: T. Kawai, C. G. Wang. *email: gqzheng123@gmail.com
} 
E⿳亠口冋口十 xploring topological materials and their electronic functions are among the front-most topics of current condensed matter physics. In particular, much attention has been paid in recent years to topological superconductors where Majorana fermions (excitations) are expected to appear on edges or in the vortex cores ${ }^{1,2}$. Such novel edge states can potentially be applied to fault tolerant non-Abelian quantum computing 3,4 . So far, great success has been achieved in observing the Majorana bound state on the interface of a ferromagnet or a topological insulator in proximity to an $s$-wave superconductor $^{5-8}$, or on the surface of iron-based superconductors ${ }^{9}$. In contrast, research on bulk topological superconductors progresses much more slowly. Candidates of bulk topological superconductors include superconductors with broken time reversal symmetry ${ }^{10,11}$, superconductors with broken spatial inversion symmetry 12,13 and odd-parity superconductors with spatial inversion symmetry ${ }^{14,15}$. For the last case, the criteria for topological superconductivity are effectively two fold. Namely, odd-parity of the gap function and an odd number of timereversal invariant momenta in the Brillouin zone ${ }^{14}$. Experimentally, clear evidence for odd-parity superconductivity had not been found until very recently ${ }^{16}$. Although $\mathrm{Cu}$-doped topological insulator $\mathrm{Cu}_{x} \mathrm{Bi}_{2} \mathrm{Se}_{3}{ }^{17}$ had been proposed as a candidate $^{14}$, experiments had been controversial ${ }^{18-20}$.

The discovery of spontaneous spin rotation-symmetry breaking in the bulk superconducting state of $\mathrm{Cu}_{0.3} \mathrm{Bi}_{2} \mathrm{Se}_{3}$ by nuclear magnetic resonance (NMR) measurements established the spintriplet, odd-parity superconducting state ${ }^{16}$. Since there is only one time-reversal-symmetric momentum in the Brillouin zone of $\mathrm{Cu}_{x} \mathrm{Bi}_{2} \mathrm{Se}_{3}{ }^{21}$, this material fulfills the two-fold criteria and can be classified as a topological superconductor. However, detailed gap function is still unclear. If the gap is fully-opened, then $\mathrm{Cu}_{0.3} \mathrm{Bi}_{2} \mathrm{Se}_{3}$ is a class DIII topological superconductor ${ }^{22}$, where Majorana zero-energy modes are expected at edges or vortex cores. If there are nodes in the gap function, the material is nonetheless topological just as the cases of Dirac or Weyl semimetals.

A more generally-used term associated with the gap in a spintriplet superconductor is the vector order parameter $\mathbf{d}$, whose direction is perpendicular to the direction of paired spins and whose magnitude is the gap size. The $\mathbf{d}$-vector was found to be parallel to $a$-axis (the $\mathrm{Se}-\mathrm{Se}$ bond direction) in $\mathrm{Cu}_{0.3} \mathrm{Bi}_{2} \mathrm{Se}_{3}{ }^{16}$. This was the first case where the $\mathbf{d}$-vector direction was unambiguously determined in any spin-triplet superconductor candidate. The emergent two-fold symmetry in the Knight shift below $T_{\mathrm{c}}{ }^{16}$ was interpreted by the concept of nematic order ${ }^{23}$, and had triggered many subsequent extensive studies on rotational symmetry breaking by various methods, which also revealed a two-fold symmetry in other physical properties ${ }^{24-28}$. Measurements by transport ${ }^{29}$, penetration depth ${ }^{30}$, and scanning tunneling microscope $(\mathrm{STM})^{31,32}$ suggesting unconventional superconductivity have also been reported since then.

However, why the d-vector is oriented to one of $a$-axes, and why it is robust against heat cycle through the superconducting transition, remain unknown. Note that there are three equivalent $a$-axis directions. This issue is important as the gap symmetry ("nematicity" indicator) is closely tied to the direction of the dvector $^{23}$. From material point of view, it had been unclear whether the carrier density can be controlled and how the physical properties would change with changing carrier density. A previous report showed that the Hall coefficient does not change even though the nominal $x$ increases from 0.15 to $0.45^{33}$. These are the issues we wish to address in this article.

In this work, we synthesized $\mathrm{Cu}$-doped $\mathrm{Bi}_{2} \mathrm{Se}_{3}$ single crystals by the electrochemical intercalating method. Through the measurements of the Knight shift, we find unprecedentedly that the carrier concentration further increases with increasing $x$ beyond $x=0.37$. We study the angle dependence of the upper critical field $H_{\mathrm{c} 2}$ in different crystals. For an odd-parity gap function, the gap anisotropy will lead to an anisotropic $H_{\mathrm{c} 2}$. One then can obtain knowledge about how the superconducting gap evolves with $x$ by measuring the $H_{\mathrm{c} 2}$ anisotropy. For samples with $x=$ $0.28,0.36$, and 0.37 which have the same size of the Knight shift, we find a two-fold symmetry in the in-plane $H_{\mathrm{c} 2}$ by ac susceptibility and magnetoresistance measurements, in agreement with previous reports ${ }^{16,24-28}$. However, the angle at which $H_{\mathrm{c} 2}$ is a minimum differs by $90^{\circ}$, which means that the direction of the dvector is different for each crystal. In contrast, for $x=0.46$ and 0.54 , two-fold anisotropy disappears, which indicates a nematicto-isotropic transition of the gap symmetry as carrier density increases. We discuss possible exotic (chiral) superconducting state for the samples with large $x$.

\section{Results}

Sample characterization. Figure 1 shows the result of dc susceptibility measurements for representative samples. In Fig. 2, we summarize the properties of all the samples we synthesized. The obtained $T_{\mathrm{c}}$ and shielding fraction (SF) for most samples are close to the values reported by Kriener et $\mathrm{al}^{34}$. The SF for $x=0.46$ is the highest (56.2\%) among those reported so far. In Table 1 we list the properties for the five samples that we will discuss in this paper.

Figure 3a shows the ${ }^{77} \mathrm{Se}(I=1 / 2)$-NMR spectra above $T_{\mathrm{c}}$ for five samples with different $x$. Each spectrum can be fitted by a single Gaussian. The Knight shift is $\sim 0.048 \%$ for $x=0.28,0.36$, and 0.37 , which is close to the value $(K=0.049 \%)$ previously reported for the sample with $x=0.3^{16}$. On the other hand, the Knight shift increased substantially in the samples with $x=0.46$ and 0.54. Generally, the Knight shift is expressed as,

$$
\begin{gathered}
K=K_{\text {orb }}+K_{\mathrm{s}}, \\
K_{\mathrm{s}}=A_{\mathrm{hf}} \chi_{\mathrm{s}},
\end{gathered}
$$

where $K_{\mathrm{orb}}$ is the contribution due to orbital susceptibility and $A_{\mathrm{hf}}$ is the hyperfine coupling constant, which are independent of carrier density. The $\chi_{\mathrm{s}}$ is the spin susceptibility which is proportional to electronic density of states. For this field configuration, $K_{\text {orb }}=0.03 \%^{16}$. In Fig. $3 b$, we plotted the Knight shift as a function of $\mathrm{Cu}$ content $x$. Upon doping, $K$ increases as compared to the parent compound, which implies that the carrier is indeed doped into the sample. The fact that $K$ has a similar value for the samples with $x=0.28-0.37$ indicates that the carrier density does not change appreciably in this $x$ region. Such behavior is consistent with other reports by different methods 33,35 . On the other hand, in the high- $x$ region, we discovered for the first time that carriers increase further as $x$ increases beyond 0.37 .

We briefly comment on possible mechanisms of $\mathrm{Cu}$-doping. In Sr-doped system ${ }^{35}, \mathrm{Sr}$ goes into multiple sites, namely, the intercalated site in-between the quintuple layer blocks, interstitial site, or the in-plane site substituting for $\mathrm{Bi}$. $\mathrm{Cu}(\mathrm{Sr})$ residing on the intercalated or interstitial site contributes electrons, while $\mathrm{Cu}(\mathrm{Sr})$ going to the substituting site contributes holes ${ }^{17}$. The stage-like $x$ dependence of $K$ suggests that $\mathrm{Cu}$ doping mechanism in each $x$ range is not simple, but rather $\mathrm{Cu}$ may also go into multiple sites. As post annealing is necessary after electrochemical process in the present case, we speculate that $\mathrm{Cu}$ may migrate into a different site after annealing, resulting in the peculiar behavior of $K$ with respect to $x$. The $\mathrm{Cu}$ position is one of the issues that needs to be addressed in future works. 


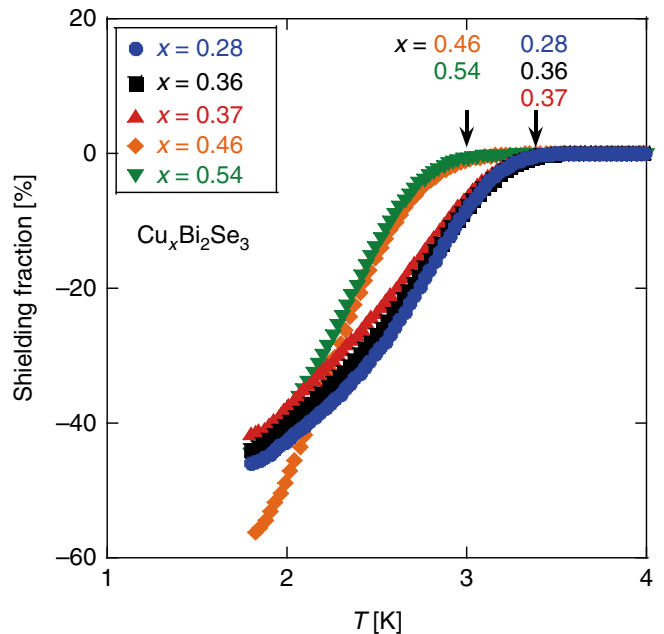

Fig. 1 Diamagnetism measurements. Shielding fraction for five samples with different $x$. The arrows indicate $T_{\mathrm{c}}$.

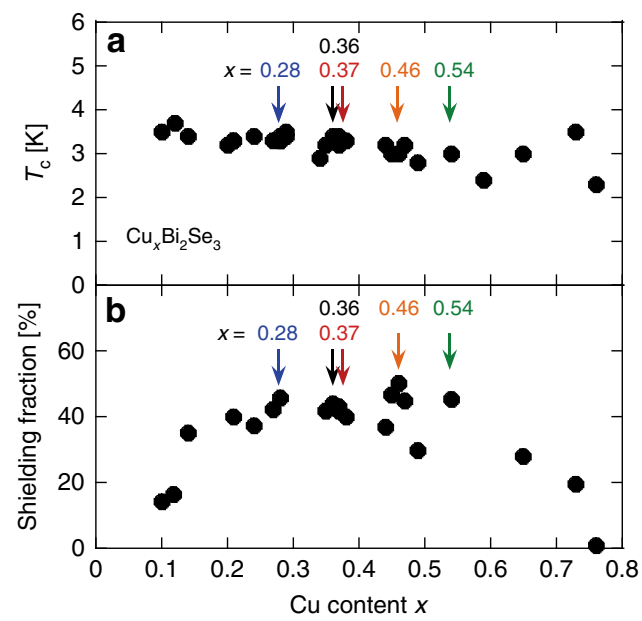

Fig. 2 Properties of the samples. Cu content (x) dependence of (a) the superconducting critical temperature $T_{\mathrm{c}}$ and $(\mathbf{b})$ the shielding fraction at $1.8 \mathrm{~K}$. The arrows indicate samples used for the $H_{\mathrm{c} 2}$ and NMR measurements reported in this paper.

\begin{tabular}{|c|c|c|c|c|c|}
\hline Cu content $x$ & 0.28 & 0.36 & 0.37 & 0.46 & 0.54 \\
\hline$T_{\mathrm{c}}[\mathrm{K}]$ & 3.4 & 3.4 & 3.4 & 3.0 & 3.0 \\
\hline Shielding fraction [\%] & 45.9 & 44.1 & 41.5 & 56.2 & 44.7 \\
\hline
\end{tabular}

Anisotropy of the upper critical field $\boldsymbol{H}_{\mathrm{c} 2}$ and its disappearance. Next we present data on the anisotropy of $H_{\mathrm{c} 2}$. For $x=0.28$, we measured both ac susceptibility (ac- $\chi$ ) and electrical resistance by changing the magnetic field for each field-direction relative to $a$ axis. The inset to Fig. 4 a shows the angle $\phi$ between the applied magnetic field $H$ and the $a$-axis. Before $\mathrm{Cu}$ doping, the direction of $a$-axis (Se-Se bond direction) was determined by Laue diffraction, which we assign as $\phi=0^{\circ}$. The main panel of Fig. $4 \mathrm{a}$ shows the magnetic field dependence of the ac- $\chi$ at different $\phi$ for $x=0.28$. As can be seen in the figure, the field dependence of ac- $\chi$ a
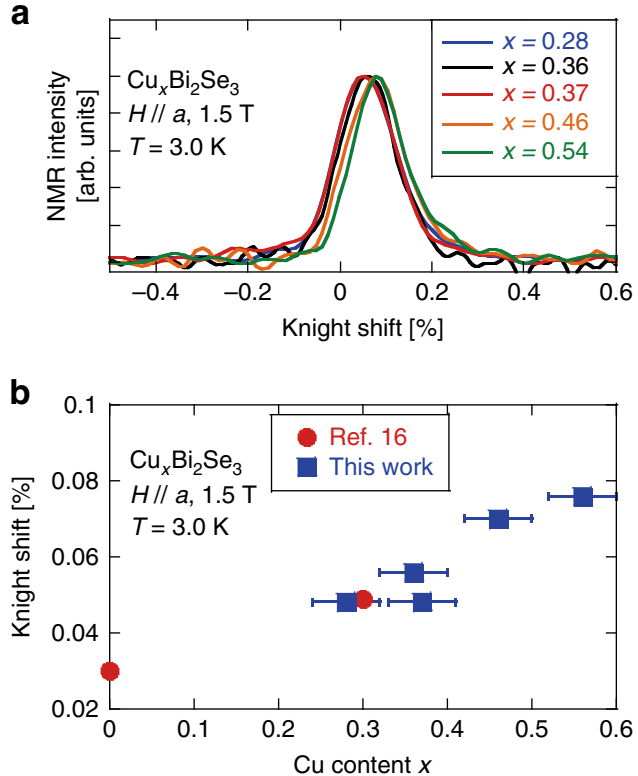

Fig. 3 NMR Spectra and Knight shift. a The ${ }^{77}$ Se-NMR spectra measured in a magnetic field of $H_{0}=1.5 \mathrm{~T}$. $\mathbf{b}$ Cu content $x$ dependence of the Knight shift. Data for $x=0$ and 0.3 are from ref. ${ }^{16}$. The error for $\mathrm{Cu}$-content $x$ was determined by the resolution of the electronic balance used for measuring the weight of samples.

is clearly different for each angle below a certain field $\left(H_{\mathrm{c} 2}\right)$, while above $H_{\mathrm{c} 2}$ the $H$-dependence of ac- $\chi$ is the same for all angles. Fig. $4 \mathrm{~b}$ shows $H_{\mathrm{c} 2}$ as a function of angle. A clear two-fold symmetry is observed.

Such two-fold symmetry is also seen in the magnetoresistance measurements, as demonstrated in Fig. 5 where the electrical resistance under a field of $0.9 \mathrm{~T}$ and at $T=1.8 \mathrm{~K}$ is plotted as a function of angle. We checked the angle-resolved resistance at $\mu_{0} H=3 \mathrm{~T}$ and $T=1.8 \mathrm{~K}$ where the sample is in the normal state and found only a random noise with an amplitude no bigger than $0.1 \mathrm{~m} \Omega$, thus confirming that such two-fold oscillation in the resistance shown in Fig. $5 b$ is caused by the anisotropy of $H_{\text {c2. }}$. Fig. 6a shows the resistance as a function of magnetic field at $T=1.8 \mathrm{~K}$ for three representative angles. Similar to the results shown in Fig. 4a, a clear angle dependence is found. Fig. 6b shows the angle dependence of $H_{\mathrm{c} 2}^{\rho}$ obtained from the magnetoresistance data. A two-fold symmetry is also clearly seen. This result is in agreement with that seen in the ac- $\chi$ data shown in Fig. 4 b, although such-defined $H_{c 2}^{\rho}$ is slightly higher but is not surprising for its definition. An in-plane anisotropy of $H_{\mathrm{c} 2}$ is also resolved from the data of electrical resistance $v s$ temperature under different magnetic fields (see Supplementary Figs. 1, 2).

Quite often, extracting $H_{\mathrm{c} 2}$ from the magnetic susceptibility has several advantages over that from magnetoresistance measurements. Firstly, the magnetic susceptibility is more sensitive to superconducting volume fraction rather than surface. Secondly, in two-dimensional or layered superconductors, $H_{\mathrm{c} 2}$ determined by resistivity measurements is often inaccurate because of vortex lattice melting ${ }^{36}$. For example, in high- $T_{\mathrm{c}}$ cuprates, even in the resistive state, one is still in a regime dominated by Cooper pairings in the presence of vortices ${ }^{36}$. For these reasons and the technical merit that ac- $\chi$ can be measured to a lower temperature in our case, below we discuss the evolution of gap symmetry based on the ac- $\chi$ data. Data for $x=0.36,0.37,0.46$, and 0.54 are shown in Fig. 7. For $x=0.36$ and 0.37 , clear angle dependence was found as in $x=0.28$. In contrast, the tendency is completely 

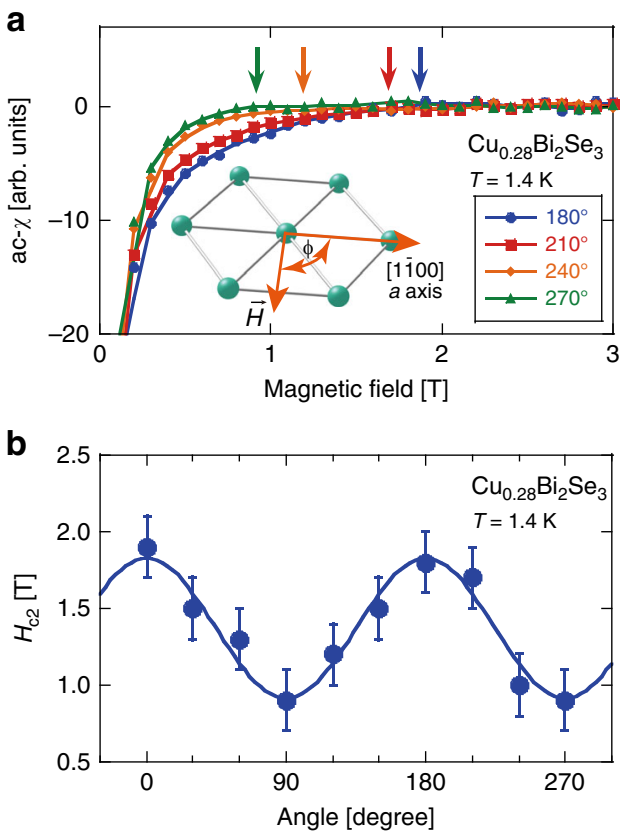

Fig. 4 In-plane upper critical field anisotropy determined by magnetic susceptibility. a Magnetic field dependence of the ac- $\chi$ at different in-plane angle $\phi$ for the sample with $x=0.28$. The solid curves are guides to the eyes. The arrows indicate $H_{\mathrm{c} 2}$ for each angle, which is defined as a point off the straight line drawn from high-field value (the normal state). The error was estimated by assuming that the uncertainty equals twice point-interval in measuring the ac- $\chi$ vs field curve. For more details see Supplementary Information. The inset is an illustration depicting the hexagonal plane and the angle $\phi$. $\mathbf{b} H_{\mathrm{c} 2}$ plotted as a function of in-plane angle $\phi$. The blue curve is a sine function.
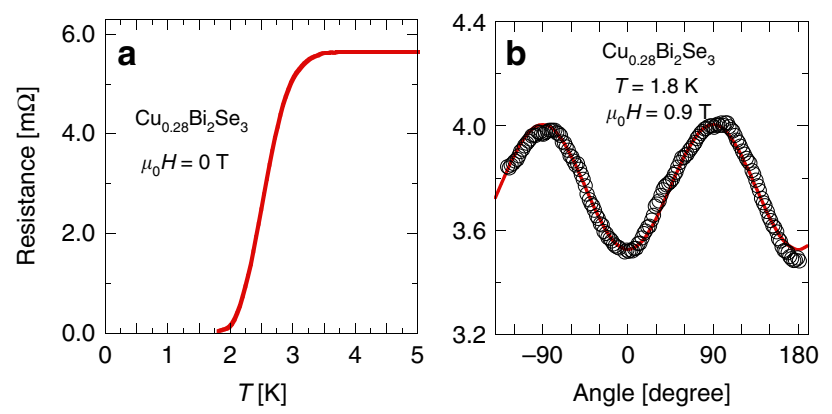

Fig. 5 Magneto-resistance measurements. a Temperature dependence of the electrical resistance at zero magnetic field, which is in agreement with ref. ${ }^{34}$. b Electrical resistance under a magnetic field of $0.9 T$ and at $T=$ $1.8 \mathrm{~K}$ as a function of in-plane angle $\phi$. Note that a minimum in the electrical resistance corresponds to a maximal $H_{\mathrm{c} 2}$. The red curve is a sine function.

different for samples with larger $x$. There is no angle dependence in ac- $\chi$ for the samples with $x=0.46$ and 0.54 .

The angular dependence of $H_{\mathrm{c} 2}$ at $1.4 \mathrm{~K}$ determined from the ac- $\chi$ for all samples is plotted in Fig. 8 (for more details, see Supplementary Fig. 3). For the samples with smaller $x$ (Fig. 8a, b), $H_{\mathrm{c} 2}$ shows a large and two-fold anisotropy. In contrast, for larger $x$ (Fig. 8c), no anisotropy is observed. The oscillation amplitude of $H_{\mathrm{c} 2}$ is similar for $x=0.28,0.36$, and 0.37 . The magnitude of $H_{\mathrm{c} 2}$ is also similar between $x=0.28$ and 0.37 , but is larger for $x=0.36$. The origin of this difference is unknown at the moment.
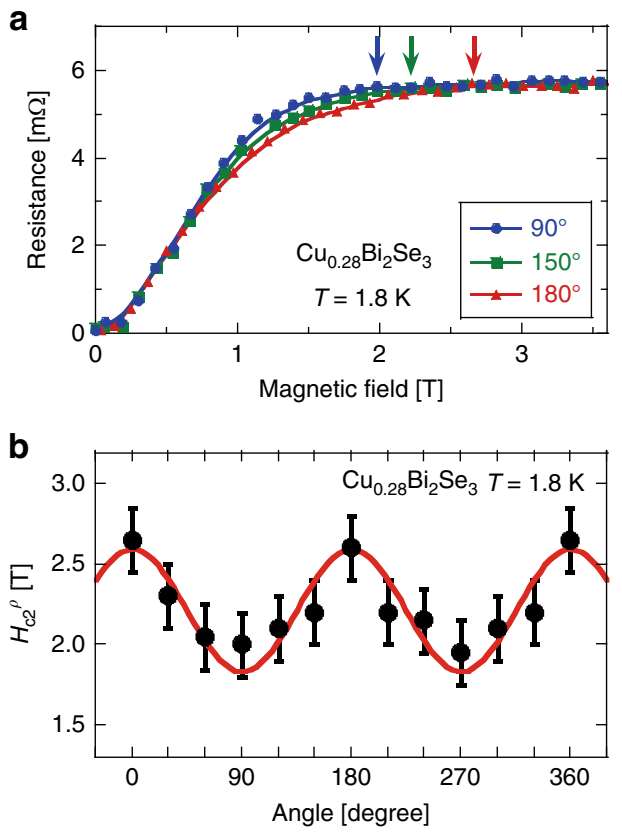

Fig. 6 In-plane upper critical field anisotropy determined by magnetoresistance. a Electrical resistance at $T=1.8 \mathrm{~K}$ as a function of the magnetic field for three representative in-plane angles. The arrows indicate $H_{c 2}$ which is defined as the field below which the data point deviates from a straight line drawn from the normal state. b Upper critical field $H_{\mathrm{c} 2}^{p}$ as defined in (a) is plotted as a function of in-plane angle. The error bar was estimated by assuming that the uncertainty equals twice point-interval in measuring the resistance vs. field curve. The red curve is a sine function.

Interestingly, the angle at which $H_{\mathrm{c} 2}$ becomes minimal is $90^{\circ}$ and $150^{\circ}$ (perpendicular to Se-Se bond) for $x=0.28$ and 0.36 , respectively, but is $0^{\circ}$ (along Se-Se bond) for $x=0.37$. For this crystal structure, the equivalent crystal-axis direction appears every $60^{\circ}$. If two oscillation patterns have a phase difference of $60^{\circ}$, it can be said that they are the same crystallographically. Therefore, the samples with $x=0.28$ and 0.36 have the same gap symmetry. However, $90^{\circ}$ difference means that the gap symmetry is different. It is noted that the angle at which $H_{\mathrm{c} 2}$ is minimal corresponds to the direction of $\mathbf{d}$-vector ${ }^{16}$. Therefore, our result indicates that the d-vector direction differs for each sample in $\mathrm{Cu}_{x} \mathrm{Bi}_{2} \mathrm{Se}_{3}$ even though the carrier density is the same or very similar. The $x=0.37$ sample has the same symmetry as the previous sample used for NMR measurement where the $\mathbf{d}$-vector is pinned to Se-Se direction ${ }^{16}$, while the $x=0.28$ and 0.36 samples have the same symmetry as the sample used for specific heat measurements ${ }^{24}$.

No multiple domains. As for the disappearance of oscillation in $H_{\mathrm{c} 2}$ for $x=0.46$ and 0.54 , a possibility of multiple domains each of which is a nematic phase with $\mathbf{d}$-vector pointing to a different direction, can be ruled out. In that case, the NMR spectrum for $T<T_{\mathrm{c}}$ would be broadened compared to that at $T>T_{\mathrm{c}}$. This is because the spectrum coming from the domains with $H \| \mathbf{d}$-vector will shift to a lower frequency ${ }^{16}$, but that from domains with $H \perp$ d-vector will not. However, our result (Fig. 9a-c) shows no such broadening which is not compatible with the multiple-domains scenario. In Fig. 9c, we show a simulation for the spectrum in case of multiple domains, assuming that various domains are distributed randomly as to wipe out oscillations in the angle dependence of $H_{\mathrm{c} 2}$. The simulation also assumed that, for $H \| \mathbf{d}$ vector, the spin Knight shift $\left(K_{\mathrm{s}}\right)$ below $T_{\mathrm{c}}$ follows the same temperature dependence as found in $\mathrm{Cu}_{0.3} \mathrm{Bi}_{2} \mathrm{Se}_{3}{ }^{16} ; K_{\mathrm{s}}=0.046 \%$ 

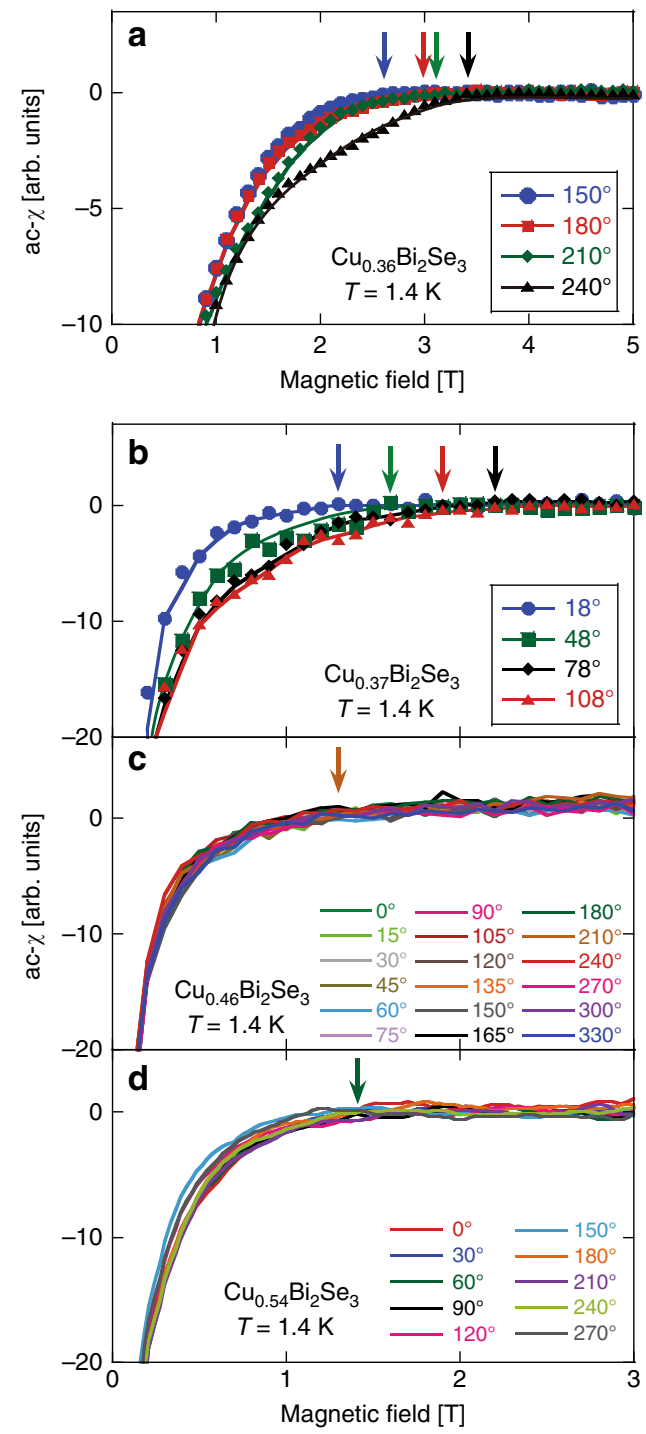

Fig. 7 Magnetic field dependence of the ac- $\chi$ at different angle $\phi$ for $x=$ $\mathbf{0 . 3 6}, \mathbf{0 . 3 7}, \mathbf{0 . 4 6}$, and $\mathbf{0 . 5 4}$. The solid curves are guides to the eyes. For sake of clarity, the data points for $x=0.46$ and 0.54 are represented by symbols with minimized size. The arrows indicate $H_{\mathrm{c} 2}$ defined in the same way as previous figures. For $x=0.46$ and 0.54 , the arrow is for $\phi=15$ and $90^{\circ}$, respectively.

and the reduction of $K_{\mathrm{s}}$ at $T=1.5 \mathrm{~K}, \Delta K_{\mathrm{s}}=0.043 \%$, were used in the simulation.

\section{Discussion}

Fu pointed out that the spin rotation-symmetry breaking (the inplane Knight shift nematicity) can be understood if the pairing function is a doublet representation ${ }^{23}, \Delta_{4 x}$ or $\Delta_{4 y}$, both of which being $p$-wave. For $\Delta_{4 x}$ state, the $\mathbf{d}$-vector is along the principle crystal axis while it is orthogonal to the principle crystal axis for $\Delta_{4 y}$ state. In the $\Delta_{4 x}$ state, there are two point nodes in the superconducting gap, which are in the direction perpendicular to the Se-Se bond. In the $\Delta_{4 y}$ state, there is no node but a minimum in the superconducting gap, and the d-vector is oriented perpendicular to the gap-minimum direction. Applying Fu's theory to our results, the $x=0.37$ and the previous $x=0.3^{16}$ samples would correspond to $\Delta_{4 x}$, and the samples with $x=0.28$ and 0.36 would correspond to $\Delta_{4 y}$ state.
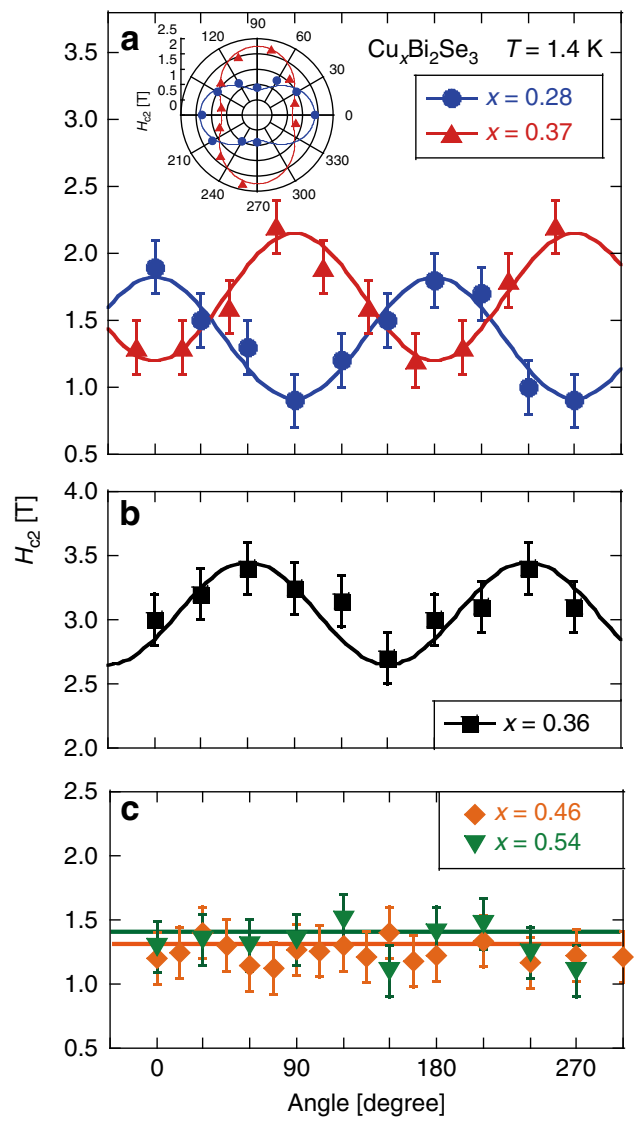

Fig. 8 In-plane $\boldsymbol{H}_{\mathrm{c} 2}$ at $T=1.4 \mathrm{~K}$ extracted from ac- $\chi$ as a function of angle $\boldsymbol{\phi}$ for all samples. The error bar was estimated by assuming that the uncertainty equals twice point-interval in measuring the ac- $\chi$ vs field curve. The solid curves are sine functions. The inset to (a) is a polar plot of the angular dependence $H_{\mathrm{c} 2}$ for $x=0.28$ and 0.37 .

As mentioned in ref. ${ }^{23}$, the theory does not take crystalline anisotropy into consideration so that $\Delta_{4 x}$ and $\Delta_{4 y}$ are degenerate. In real crystals, however, crystalline distortions due to dopants exist, and it was suggested that the dopant position is important for superconductivity 35,37 . Hexagonal distortion was indeed reported in $\mathrm{Sr}_{x} \mathrm{Bi}_{2} \mathrm{Se}_{3}{ }^{38}$. The same can be expected in $\mathrm{Cu}_{x} \mathrm{Bi}_{2} \mathrm{Se}_{3}{ }^{39}$. Moreover, strains induced by quenching can vary from one sample to another. The chemical processes to obtain a sample with high SF are complex which includes a quenching process. Only those quenched from a narrow temperature $\left(\sim 560^{\circ} \mathrm{C}\right)$ show high SF, which suggests that it is important to seize a metallurgically meta-stable phase to obtain the superconductivity. However, the quenching process is less controllable so that strain induced during this process is random among samples. We believe that $\mathbf{d}$-vector pointing to different directions in samples with the same carrier density is due to a different local structural environment such as strain caused by quenching, dopant-induced crystal distortion, etc.

The most intriguing and surprising finding of this work is that the oscillation in $H_{\mathrm{c} 2}$ disappears for the samples with $x=0.46$ and 0.54 , as seen in Fig. 8 b. Judging from the NMR spectrum width which is almost the same for $x=0.36,0.48$, and 0.54 in particular, we conclude that the sample homogeneity is very similar among them. Also, a multiple-domains scenario can be ruled out as discussed in the previous section. We interpret the surprising evolution of $H_{\mathrm{c} 2}$ as due to an emergent fully-opened isotropic gap for large $x$. It was previously pointed out that the odd-parity superconductivity with two-component $E_{\mathrm{u}}$ representation admits 

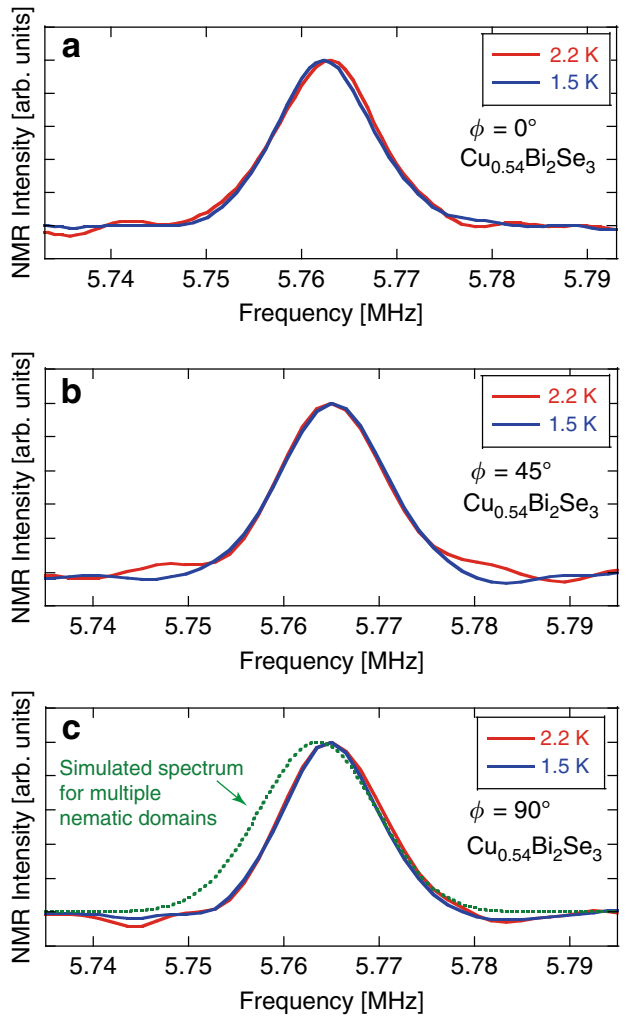

Fig. 9 NMR spectra and simulation. NMR spectra at $T_{\mathrm{c}}(H=0.7 \mathrm{~T})=2.2 \mathrm{~K}$ and below for $x=0.54$ with different angles between $H$ and the $a$-axis (a-c). c A simulation for the spectrum in presence of multiple nematic domains, simply assuming that various domains are distributed randomly.

two possible phases, nematic and chiral ${ }^{40,41}$. Under some circumstances, a chiral, fully-gapped, state becomes more stable as compared to a nematic state. One of the crucial parameters important for selecting between the two states is the Fermi surface shape. It has been theoretically shown by several groups that the chiral state can be stabilized when the Fermi surface becomes more two-dimensional ${ }^{42-44}$. Experimentally, photoemission and quantum oscillation measurements have suggested that the Fermi surface becomes more two-dimensional as carrier density increases ${ }^{45,46}$. Indeed, the Knight shift result indicates that carriers increased in the samples of $x=0.46$ and 0.54 as compared to $x \leq$ 0.37 . Therefore, the dramatic change in the angle dependence of the $H_{\mathrm{c} 2}$ can be naturally explained as due to a gap symmetry change from nematic to chiral. The lack of change of the Knight shift below $T_{\mathrm{c}}$ for all angles (Fig. 9) as contrary to what found in the nematic phase ${ }^{16}$ is consistent with existing theory that the $\mathbf{d}$ vector for the chiral phase would be pointing to the $c$-axis ${ }^{42}$. Thus, our finding suggests that superconductors with strong spin-orbital interaction ${ }^{14}$ is a land much more fertile than we thought.

In summary, single crystal samples of $\mathrm{Cu}_{x} \mathrm{Bi}_{2} \mathrm{Se}_{3}$ with unprecedented high doping levels were newly synthesized and investigated. By NMR measurements, we found for the first time that the carrier density increased further by $\mathrm{Cu}$ doping beyond $x=$ 0.37 . By magnetic susceptibility measurements, we found that the in-plane $H_{\mathrm{c} 2}$ shows a clear two-fold oscillation for the samples with $x=0.28,0.36$, and 0.37 which have similar carrier density as evidenced by the Knight shift. However, the angle at which $H_{\mathrm{c} 2}$ becomes minimal is different by $90^{\circ}$ between different samples. This indicates that the direction of the $\mathbf{d}$-vector is different from crystal to crystal due to a different local structure caused by strain during the quenching process, dopant-induced crystal distortion, etc. In the samples with $x=0.46$ and 0.54 , the two-fold oscillation is completely suppressed, which indicates a gap symmetry change from nematic to isotropic as carrier density increases. These findings enriched the contents of topological superconductivity in doped $\mathrm{Bi}_{2} \mathrm{Se}_{3}$, and we hope that our work will stimulate further studies on possibly even more exotic superconducting state (possible chiral state) in the high-doped region of $\mathrm{Cu}_{x} \mathrm{Bi}_{2} \mathrm{Se}_{3}$ as well as on bulk topological superconductors in general.

\section{Methods}

Single crystal growth and characterization. Single crystals of $\mathrm{Cu}_{x} \mathrm{Bi}_{2} \mathrm{Se}_{3}$ were prepared by intercalating $\mathrm{Cu}$ into $\mathrm{Bi}_{2} \mathrm{Se}_{3}$ by the electrochemical doping method described in ref. ${ }^{34}$. First, single crystals of $\mathrm{Bi}_{2} \mathrm{Se}_{3}$ were grown by melting stoichiometric mixtures of elemental Bi (99.9999\%) and Se $(99.999 \%)$ at $850{ }^{\circ} \mathrm{C}$ for $48 \mathrm{~h}$ in sealed evacuated quartz tubes. After melting, the sample was slowly cooled down to $550^{\circ} \mathrm{C}$ over $48 \mathrm{~h}$ and kept at the same temperature for $24 \mathrm{~h}$. Those meltgrown $\mathrm{Bi}_{2} \mathrm{Se}_{3}$ single crystals were cleaved into smaller rectangular pieces of about $14 \mathrm{mg}$. They were wound by bare copper wire (dia. $0.05 \mathrm{~mm}$ ), and used as a working electrode. A $\mathrm{Cu}$ wire with diameter of $0.5 \mathrm{~mm}$ was used both as the counter (CE) and the reference electrode (RE). We applied a current of $10 \mu \mathrm{A}$ in a saturated solution of $\mathrm{CuI}$ powder $(99.99 \%)$ in acetonitrile $\left(\mathrm{CH}_{3} \mathrm{CN}\right)$. The obtained crystals samples were then annealed at $560^{\circ} \mathrm{C}$ for $1 \mathrm{~h}$ in sealed evacuated quartz tubes, and quenched into water. After quenching, the samples were covered with epoxy (STYCAST 1266) to avoid deterioration. The Cu concentration $x$ was determined from the mass increment of the samples. To check the superconducting properties, dc susceptibility measurements were performed using a superconducting quantum interference device (SQUID) with the vibrating sample magnetometer (VSM).

NMR measurements. The ${ }^{77} \mathrm{Se}-\mathrm{NMR}$ spectra were obtained by the fast Fourier transformation of the spin-echo at a fixed magnetic field (1.5 T or 0.7 T). The Knight shift $K$ was calculated using nuclear gyrometric ratio $\gamma_{\mathrm{N}}=8.118 \mathrm{MHz} / \mathrm{T}$ for ${ }^{77} \mathrm{Se}$.

Angle-resolved $\boldsymbol{H}_{\mathbf{c} 2}$ measurements. $H_{\mathrm{c} 2}$ was determined from ac susceptibility by measuring the inductance of in-situ NMR coil. Angle-dependent measurements were performed by using a piezo-driven rotator (Attocube ANR51) equipped with Hall sensors to determine the angle between magnetic field and crystal axis. The ac$\chi$ vs. $H$ data in the normal state were fitted by a linear function (a constant line). $H_{\mathrm{c} 2}$ was defined as a point off the straight line. A typical example is shown in Supplementary Fig. 3 .

Magnetoresistance measurements. The angle-dependent electrical resistance was measured by the standard four-electrode method in a physical properties measurement system (PPMS, Quantum Design) with a mechanical rotating probe. The building of electrodes were carried out in a glove box filled with high-purity Ar gas to prevent sample from degradation. The electrodes were made such that the current direction is along the $a$-axis. The excitation currents are $0.1-1 \mathrm{~mA}$ to make a compromise of the Joule heating and the measurement accuracy.

\section{Data availability}

The data that support the findings of this study are available on reasonable request.

Received: 5 April 2019; Accepted: 18 December 2019;

Published online: 13 January 2020

\section{References}

1. Qi, X.-L. \& Zhang, S.-C. Topological insulators and superconductors. Rev. Mod. Phys. 83, 1057-1110 (2011).

2. Sato, M. \& Ando, Y. Topological superconductors: a review. Rep. Prog. Phys. 80, 076501 (2017).

3. Freedman, M. H., Kitaev, A., Larsen, M. J. \& Wang, Z. Topological quantum computation. Bull. Am. Math. Soc. 40, 31-38 (2003).

4. Kitaev, A. Fault-tolerant quantum computation by anyons. Ann. Phys. 303, 2 (2003).

5. Fu, L. \& Kane, C. L. Superconducting proximity effect and Majorana Fermions at the surface of a topological insulator. Phys. Rev. Lett. 100, 096407 (2008).

6. Mourik, V. et al. Signatures of Majorana Fermions in hybrid superconductorsemiconductor nanowire devices. Science 336, 1003-1007 (2012).

7. Nadj-Perge, S. et al. Observation of Majorana fermions in ferromagnetic atomic chains on a superconductor. Science 346, 602-607 (2014).

8. Sun, H.-H. et al. Majorana zero mode detected with spin selective andreev reflection in the vortex of a topological superconductor. Phys. Rev. Lett. 116, 257003 (2016) 
9. Wang, D. et al. Evidence for Majorana bound states in an iron-based superconductor. Science 362, 333-335 (2018).

10. Senthil, T., Marston, J. B. \& Fisher, M. P. A. Spin quantum Hall effect in unconventional superconductors. Phys. Rev. B 60, 4245-4254 (1999).

11. Wilczek, F. Majorana returns. Nat. Phys. 5, 614 (2009).

12. Nishiyama, M., Inada, Y. \& Zheng, G.-q Spin triplet superconducting state due to broken inversion symmetry in $\mathrm{Li}_{2} \mathrm{Pt}_{3} \mathrm{~B}$. Phys. Rev. Lett. 98, 047002 (2007).

13. Sato, M. \& Fujimoto, S. Topological phases of noncentrosymmetric superconductors: edge states, majorana fermions, and non-Abelian statistics. Phys. Rev. B 79, 094504 (2009).

14. Fu, L. \& Berg, E. Odd-parity topological superconductors: theory and application to $\mathrm{Cu}_{x} \mathrm{Bi}_{2} \mathrm{Se}_{3}$. Phys. Rev. Lett. 105, 097001 (2010).

15. Sato, M. Topological odd-parity superconductors. Phys. Rev. B 81, 220504 (2010).

16. Matano, K., Kriener, M., Segawa, K., Ando, Y. \& Zheng, G.-q Spin-rotation symmetry breaking in the superconducting state of $\mathrm{Cu}_{x} \mathrm{Bi}_{2} \mathrm{Se}_{3}$. Nat. Phys. 12, 852 (2016)

17. Hor, Y. S. et al. Superconductivity in $\mathrm{Cu}_{x} \mathrm{Bi}_{2} \mathrm{Se}_{3}$ and its implications for pairing in the undoped topological insulator. Phys. Rev. Lett. 104, 057001 (2010).

18. Sasaki, S. et al. Topological superconductivity in $\mathrm{Cu}_{x} \mathrm{Bi}_{2} \mathrm{Se}_{3}$. Phys. Rev. Lett. 107, 217001 (2011).

19. Peng, H., De, D., Lv, B., Wei, F. \& Chu, C.-W. Absence of zero-energy surface bound states in $\mathrm{Cu}_{x} \mathrm{Bi}_{2} \mathrm{Se}_{3}$ studied via Andreev reflection spectroscopy. Phys. Rev. B 88, 024515 (2013).

20. Levy, N. et al. Experimental evidence for $s$-wave pairing symmetry in superconducting $\mathrm{Cu}_{x} \mathrm{Bi}_{2} \mathrm{Se}_{3}$ single crystals using a scanning tunneling microscope. Phys. Rev. Lett. 110, 117001 (2013).

21. Wray, L. et al. Observation of unconventional band topology in a superconducting doped topological insulator, $\mathrm{Cu}_{x}-\mathrm{Bi}_{2} \mathrm{Se}_{3}$ : topological superconductor or non-Abelian superconductor? arXiv:0912.3341 (2009).

22. Schnyder, A. P., Ryu, S., Furusaki, A. \& Ludwig, A. W. W. Classification of topological insulators and superconductors in three spatial dimensions. Phys. Rev. B 78, 195125 (2008).

23. Fu, L. Odd-parity topological superconductor with nematic order: application to $\mathrm{Cu}_{x} \mathrm{Bi}_{2} \mathrm{Se}_{3}$. Phys. Rev. B 90, 100509 (2014).

24. Yonezawa, S. et al. Thermodynamic evidence for nematic superconductivity in $\mathrm{Cu}_{\mathrm{x}} \mathrm{Bi}_{2} \mathrm{Se}_{3}$. Nat. Phys. 13, 123 (2017).

25. Pan, Y. et al. Rotational symmetry breaking in the topological superconductor $\mathrm{Sr}_{x} \mathrm{Bi}_{2} \mathrm{Se}_{3}$ probed by upper-critical field experiments. Sci. Rep. 6, 28632 (2016).

26. Nikitin, A. M., Pan, Y., Huang, Y. K., Naka, T. \& de Visser, A. High-pressure study of the basal-plane anisotropy of the upper critical field of the topological superconductor $\mathrm{Sr}_{x} \mathrm{Bi}_{2} \mathrm{Se}_{3}$. Phys. Rev. B 94, 144516 (2016).

27. Asaba, T. et al. Rotational symmetry breaking in a trigonal superconductor Nb-doped $\mathrm{Bi}_{2} \mathrm{Se}_{3}$. Phys. Rev. X 7, 011009 (2017).

28. Du, G. et al. Superconductivity with two-fold symmetry in topological superconductor $\mathrm{Sr}_{\mathrm{x}} \mathrm{Bi}_{2} \mathrm{Se}_{3}$. Sci. China Phys. Mech. Astron. 60, 037411 (2017).

29. Smylie, M. P. et al. Evidence of nodes in the order parameter of the superconducting doped topological insulator $\mathrm{Nb}_{x} \mathrm{Bi}_{2} \mathrm{Se}_{3}$ via penetration depth measurements. Phys. Rev. B 94, 180510 (2016).

30. Smylie, M. P. et al. Robust odd-parity superconductivity in the doped topological insulator $\mathrm{Nb}_{x} \mathrm{Bi}_{2} \mathrm{Se}_{3}$. Phys. Rev. B 96, 115145 (2017).

31. Sirohi, A. et al. Low-energy excitations and non-BCS superconductivity in $\mathrm{Nb}_{x} \mathrm{Bi}_{2} \mathrm{Se}_{3}$. Phys. Rev. B 98, 094523 (2018).

32. Tao, R. et al. Direct visualization of the nematic superconductivity in $\mathrm{Cu}_{x} \mathrm{Bi}_{2} \mathrm{Se}_{3}$. Phys. Rev. X 8, 041024 (2018).

33. Kriener, M., Segawa, K., Sasaki, S. \& Ando, Y. Anomalous suppression of the superfluid density in the $\mathrm{Cu}_{x} \mathrm{Bi}_{2} \mathrm{Se}_{3}$ superconductor upon progressive $\mathrm{Cu}$ intercalation. Phys. Rev. B 86, 180505 (2012).

34. Kriener, M. et al. Electrochemical synthesis and superconducting phase diagram of $\mathrm{Cu}_{x} \mathrm{Bi}_{2} \mathrm{Se}_{3}$. Phys. Rev. B 84, 054513 (2011).

35. $\mathrm{Li}, \mathrm{Z}$. et al. Possible structural origin of superconductivity in $\mathrm{Sr}$-doped $\mathrm{Bi}_{2} \mathrm{Se}_{3}$. Phys. Rev. Mater. 2, 014201 (2018).

36. Ramshaw, B. J. et al. Vortex lattice melting and $H_{c 2}$ in underdoped $\mathrm{YBa}_{2} \mathrm{Cu}_{3}$ Oy. Phys. Rev. B 86, 174501 (2012).

37. Schneeloch, J. A., Zhong, R. D., Xu, Z. J., Gu, G. D. \& Tranquada, J. M. Dependence of superconductivity in $\mathrm{Cu}_{\mathrm{x}} \mathrm{Bi}_{2} \mathrm{Se}_{3}$ on quenching conditions. Phys. Rev. B 91, 144506 (2015).
38. Kuntsevich, A. Y. et al. Structural distortion behind the nematic superconductivity in $\mathrm{Sr}_{x} \mathrm{Bi}_{2} \mathrm{Se}_{3}$. New J. Phys. 20, 103022 (2018).

39. How, P. T. \& Yip, S.-K. Signatures of nematic superconductivity in doped $\mathrm{Bi}_{2} \mathrm{Se}_{3}$ under applied stress. Phys. Rev. B 100, 134508 (2019).

40. Venderbos, J. W. F., Kozii, V. \& Fu, L. Odd-parity superconductors with twocomponent order parameters: nematic and chiral, full gap, and Majorana node. Phys. Rev. B 94, 180504 (2016).

41. Wu, F. \& Martin, I. Nematic and chiral superconductivity induced by odd parity fluctuations. Phys. Rev. B 96, 144504 (2017).

42. Uematsu, H., Mizushima, T., Tsuruta, A., Fujimoto, S. \& Sauls, J. A. Chiral Higgs mode in nematic superconductors. Phys. Rev. Lett. 123, 237001 (2019).

43. Chirolli, L. Chiral superconductivity in thin films of doped $\mathrm{Bi}_{2} \mathrm{Se}_{3}$. Phys. Rev. B 98, 014505 (2018).

44. Yang, L. \& Wang, Q.-H. The direction of the $d$-vector in a nematic triplet superconductor. New J. Phys. 21, 093036 (2019).

45. Lahoud, E. et al. Evolution of the Fermi surface of a doped topological insulator with carrier concentration. Phys. Rev. B 88, 195107 (2013).

46. Lawson, B. J. et al. Quantum oscillations in $\mathrm{Cu}_{x} \mathrm{Bi}_{2} \mathrm{Se}_{3}$ in high magnetic fields. Phys. Rev. B 90, 195141 (2014).

\section{Acknowledgements}

We thank Y. Inada for help in Laue diffraction measurements and S. Kawasaki for help in some of the $H_{\mathrm{c} 2}$ measurements, Markus Kriener and T. Mizushima for useful discussions This work was supported in part by the JSPS/MEXT Grants (Nos. JP15H05852, JP16H04016, and JP17K14340) and MOST grant No. 2017YFA0302904.

\section{Authors contributions}

G.-q.Z. planned and supervised the project. T.Kawai, Y.K., and T.Kambe synthesized the single crystals. T.Kawai, Y.K., Y.H., and K.M. performed magnetic susceptibility, Y.H. and K.M. conducted NMR, and C.G.W. performed magneto-resistance measurements. G.-q.Z. wrote the manuscript with inputs from K.M. All authors discussed the results and interpretation.

\section{Competing interests}

The authors declare no competing interests.

\section{Additional information}

Supplementary information is available for this paper at https://doi.org/10.1038/s41467019-14126-w.

Correspondence and requests for materials should be addressed to G.-q.Z.

Peer review information Nature Communications thanks the anonymous reviewer(s) for their contribution to the peer review of this work.

Reprints and permission information is available at http://www.nature.com/reprints

Publisher's note Springer Nature remains neutral with regard to jurisdictional claims in published maps and institutional affiliations.

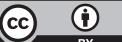

Open Access This article is licensed under a Creative Commons Attribution 4.0 International License, which permits use, sharing, adaptation, distribution and reproduction in any medium or format, as long as you give appropriate credit to the original author(s) and the source, provide a link to the Creative Commons license, and indicate if changes were made. The images or other third party material in this article are included in the article's Creative Commons license, unless indicated otherwise in a credit line to the material. If material is not included in the article's Creative Commons license and your intended use is not permitted by statutory regulation or exceeds the permitted use, you will need to obtain permission directly from the copyright holder. To view a copy of this license, visit http://creativecommons.org/ licenses/by/4.0\%

(C) The Author(s) 2020 